\title{
HAPPY OR SCARED - DETECTING EMOTIONS OF PEDELEC DRIVERS IN URBAN AREAS
}

\author{
H. Dastageeri ${ }^{1}$, P. Rodrigues ${ }^{1}$, J. Silberer ${ }^{2}$ \\ ${ }^{1}$ Dept. of Geo-Informatics, HFT - University of Applied Sciences Stuttgart, Schellingstrasse 24, Stuttgart, Germany - \\ habiburrahman.dastageeri@hft-stuttgart.de,preston.rodrigues@hft-stuttgart.de \\ ${ }^{2}$ Dept. of Business Psychology, HFT - University of Applied Sciences Stuttgart, Schellingstrasse 24, Stuttgart, Germany - \\ jan.silberer@hft-stuttgart.de
}

KEY WORDS: experience sampling, urban emotions, mobile sensors, machine learning

\begin{abstract}
:
With rising population, cities face new challenges. A key challenge for city administrators is to address the overall well-being of its citizens. This includes both physical and emotional health. Towards this objective, cities around the world are heavily investing in green mobility with support for sustainable modes (e.g. public transport, cycling, walking) as an alternative to individual motorized transport using combustion engine. However, very little attention is paid towards identifying the effect of green mobility on the emotional states of citizens. Several studies show a link between an upbeat emotional state and physical signs of good health. Furthermore, as urban centres expand it is imperative to find a balanced combination of physical and emotional health during last mile urban commute. In this paper, we try to find a feasible method for urban emotion detection in the age of last mile green mobility. Our approach relies on Machine Learning (ML) techniques to predict emotions with real-time data.
\end{abstract}

\section{INTRODUCTION}

The fast growing population of the cities forces the municipalities to react fast. According to United Nations (UN), formerly small villages like Shenzhen with 3.200 inhabitants in 1950 will have 11 million by 2025 . Furthermore, the Chinese government is planning to resettle 250 million rural population to cities within the next few years. This trend is not common to China alone, as other cities also show fast growth. For instance, Lagos grows by 67 inhabitants per hour, New Delhi by 61 and Istanbul by 46 . The situation in Europe is currently not as dire as in Asia but still significant. Berlin grows currently by five inhabitants per hour (Adli, 2017). Besides the challenges of building up a corresponding infrastructure, cities need to provide a sustainable and healthy environment. Reducing air pollution and fine dust content are becoming increasingly important. In cities where the air quality is measured, more than $80 \%$ of the inhabitants are exposed to an air pollution, which exceeds the limits of the World Health Organisation (WHO) (WHO-WHO Global Urban Ambient Air Pollution Database (update 2016), 2017). In 2012, an estimated 6.5 million deaths (11.6\% of all global deaths) were associated with indoor and outdoor air pollution together (WHO releases country estimates on air pollution exposure and health impact, 2016). It is crucial for cities to reduce air pollution and fine dust by proposing a combination of mobility solutions other than combustion engine.

Electric mobility is seen as a possible solution to tackle the ills of air pollution. All street vehicles that are powered by an electric motor and primarily get their energy from the power grid fall under this category. In addition to the vehicles, an interdependent infrastructure is needed that includes energy supply, charging and traffic management (VDI/VDE Innovation + Technik GmbH, 2016). To enhance the eco-friendly effect of the emobility, the energy source should be gained from renewable energies and contain a convenient sharing system. The variety of means of transportation and good connection to public transport can leverage the acceptance. Practical support programs and privileges such as free parking for electric cars are worthwhile for the sustainable urban mobility concept (Elektromobilität, 2019). Especially pedelecs as a mean to foster a low-carbon mobility have versatile advantages. The physical exercise for instance is one advantage that also supports wellbeing. According to the Healthy Cities Vision of WHO, health and well-being are the basic factors for urban health. A healthy city ensures that health and well-being of both the people and the planet are at the heart of all the cities internal and external policies. This includes a whole-of-city approach to health and well-being (Healthy Cities Vision, 2019). However, well-being is a subjective value. How is it possible to locate critical areas in a city, which cause stress or may harm wellbeing? Measuring emotions for creating emotional maps may be one approach and can be used as a tool for city planners (Wilhelm, Broschart and Zeile, 2015). Though measuring emotions or even the classification is a strongly discussed topic (Geven, Tscheligi and Noldus, 2009). Especially if the aim is to measure emotions out of the lab, considering the challenges of the real world outdoors. Not to mention on a mobile setting such as on a pedelec during the physical exercise of cycling.

This paper takes advantage of the recent development in the sector of sensors and Machine Learning (ML). We rely on bioand non-biosensors to gather data. Biosensors are used to measure vital bio signals while the non-biosensors measured pedelec technical data. To measure subtle bio signals various biosensors were placed directly on the body of the probands. Additionally, the pedelec was extended with a special computer unit to collect the information of the vehicle including its Global Positioning System (GPS) location. All the data was collected and merged into a single dataset during post processing. 


\section{THEORY OF EMOTIONS}

Emotions are widely debated; currently there is no consensus in the psychology community to the question of "What is/are emotion(s)?" (Beck, 2015). Moreover, there is a debate how emotional states can be measured empirically (Celeghin et al., 2017).

One of the first theories, known as the James-Lange theory, is that emotions are the experience related to the physiological responses to a stimulus (James, 1884). In this line of reasoning, the body reacts simultaneously with the emotion. This theory was followed up by many alternative theories (for an overview, see, Thanapattheerakul et al., 2018).

In our work, we consider that emotions can be predicted by measuring physiopsychological correlates. For the classification of the emotions, we follow the Core Affect which places the human state on a two or three-dimensional scale. Each scale has a level of arousal (high to low), valance (positive or negative), and at times motivation tendency (approach or avoid) (Thanapattheerakul et al., 2018).

The classification of discrete emotions are based on the schematic map of core affects by Russel and Barrett (Figure 1).

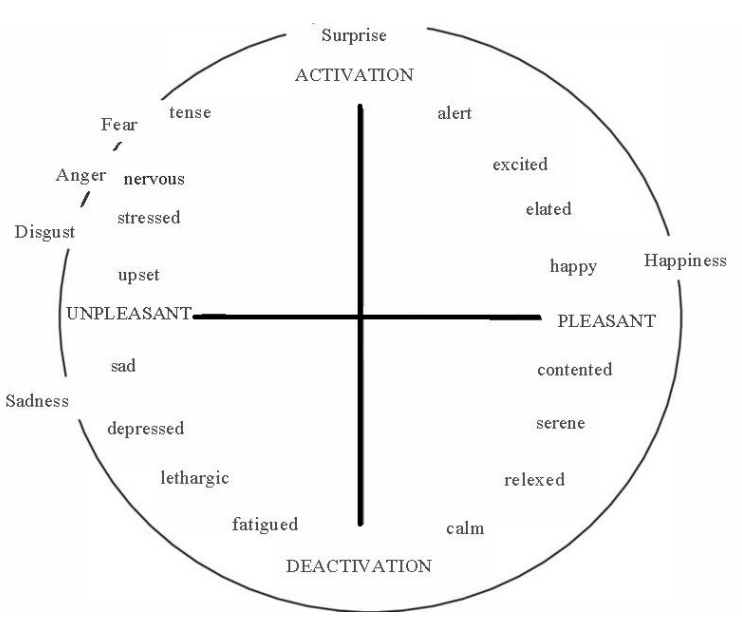

Figure 1. Classification of emotions based on the Core Affect (Barrett and Russell, 1999)

Emotional experiences are too complex to be allocated into a single scientific category. In their model Barrett and Russell (1999) thus distinguish between prototypical emotional episodes and core affect. The former refers to a set of events directed at a specific object, which the prototypical emotional episode is about (Solomon, 1976). This can be a person, condition, event or thing e.g. a person could be afraid of cycling across a large junction. Most people would probably see this as the clearest case of an emotion. Core effect on the contrary, does not have to be directed at anything (Barrett and Russell, 1999). It can be random as in moods and can become directed, when it is part of a prototypical emotional episode. For example, one could wake up in the morning feeling lethargic for no reason and this could amplify the person's fear of cycling across a large junction on that day out of the concern of being less concentrated due to this specific core affect.
In the model, core affect is represented in the inner part of the circle; prototypical emotional episodes are allocated on the outer part (Barrett and Russell, 1999). Prototypical emotional episodes vary in intensity, degree of pleasure and activation depending on the object they are directed at. For example, the fear of crossing a large junction would probably be perceived as unpleasant while the fear of cycling fast could be perceived as pleasant to a certain degree. Waking up fatigued in the morning will always be perceived as unpleasant but never as pleasant. The same logic can be applied when distinguishing prototypical emotional episodes and core affect by the degree of activation. Being happy because of the relaxing atmosphere of cycling in the park causes a different degree of physical arousal/activation than happiness caused by a fun downhill mountain bike track. Thus, the emotions on the outer circle caused by prototypical emotional episodes are less specific and cover a wider range of situations. In a first step, we focused on these in our prediction models to get an overview on which of them we can use best to make cycling more attractive for people.

\section{MEASURES}

Improving popularity for pedelecs in people by predicting emotions can be approached in different ways. On the one hand, one can try to predict when people perceive negative emotions and then find ways to avoid these situations. On the other hand, a focus on positive emotions can be set. We integrated two of the six emotions proposed by Barrett and Russell (1999): Happiness and Fear in our analysis. In doing so, we looked at one positive and one negative emotion. Fear was chosen because studies show that security concerns are one major reason for people not to cycle in the city. Happiness was chosen to also look at the positive side of well-being while riding pedelecs

We used a uni-modal method by measuring biosensors such as the heart rate (HR), heart rate variability (HRV) and skin conductance levels (SCL), skin temperature on the chest (ST) and skin temperature on the palm of hand (STH). In addition, the gender, age, height and weight were recorded.

Heart rate (HR), skin temperature and skin conductance measures are known to be suitable for the purpose of predicting emotions (Rodrigues da Silva et al. 2014). The BMI has also been adopted in previous research to predict emotions (Delgado-Rico, Elena, et al. 2012). Above that, the confounding variables age and gender have been included. Variations in physiological measures are related to age (Sammito et al. 2014) and females are known to show a stronger emotional reactivity (Rohrmann \& Hopp 2008). To measure the selected variables as precisely as possible and avoid memory distortions, experience sampling has been chosen as research approach. In this approach, participants are requested to respond to questionnaires directly after performing the behaviour of interest in the field (Csikszentmihalyi, Larson, 2014).

The biosensors were added by non-biosensors, which measured the technical components of the pedelec. This information are accessible in real time. 


\subsection{Biosensors}

\subsubsection{Heart Rate}

The HR belongs to the cardiovascular body system. HR describes the amount of blood measured in litres, which is circulated in the body per minute. According to (Siegel et al., 2018) in recent studies the HR was most frequently measured across all emotion categories to recognize an emotion. However, the HR is an ambiguous parameter. Harrison, Kreibig and Critchley (2013) for example suggested two kinds of disgust. Usually the HR goes up when one is feeling disgust. Nevertheless, in body-boundary violation, the HR goes down. For this reason, we additionally used questionnaires to assign directly emotions and minimize or prevent ambiguity.

\subsubsection{Heart Rate Variability}

The HRV as well as the HR belongs to the cardiovascular body system. The HRV measures the time between the HR in milliseconds (ms) as there is a variation. A high HRV indicates general fitness. Moreover the HRV reacts faster to changes of our body than the HR (Vesterinen et $a 1 ., 2013$ ).

\subsubsection{Skin Conductance Level}

The SCL measures the change in electrical conductance of the skin in microsiemens ( $\mu \mathrm{S})$ (Siegel et al., 2018). Due to changes in the amount of sweat on the skin the SCL changes accordingly. Hence, the SCL can correlate with arousal. Studies show that a high SCL indicates a rising level of arousal (Stockhausen, 2016).

\subsubsection{Skin Temperature on the chest/ on the palm of the hand}

The skin temperature (ST) also correlates with emotional states. A low ST indicates with a positive emotional state. The skin temperature on the palm of the hand $(\mathrm{STH})$ correlates differently. STH depends on the physical stress. Facing a low physical stress level, a low STH points towards negative emotions. Contrariwise a low STH during a high physical stress level indicates low mental stress (Kohn, 2017).

\subsection{Non-Biosensors}

\subsubsection{Measuring instruments}

The Movisens EcgMove 3 chest strap measured the HR and HRV. SLC and STH were recorded with Movisens EdaMove 3. The Garmin Fenix 5X stored the ST. On the movisensXS App using a Samsung Galaxy S5, the questionnaires were provided.

The pedelec is a smart ebike, type EB 001 from Daimler AG which was provided by our industrial partner Daimler TSS in the context of the project $i_{-}$city. The smart ebike was extended by a computer unit to provide further information of the bike. The computer unit is connected to a server, which provides the information in real time. Hence, inter alia the information of GPS, pedal force, speed, mileage and battery voltage can be fetched. The computer starts sending information as soon as the
GPS receiver detects a movement. The pedal force is an abstract unit given by the manufacturer between 0 and 40 . Speed is the current speed stated by kilometres per hour. The mileage parameter provides the total kilometres that the ebike was driven. The battery voltage informs about the current state of the battery by the familiar percental value between 0 and 100 . The data is accessible via JavaScript Object Notation (JSON) file format.

Figure 2 displays the proband on the ebike wearing all measuring instruments.

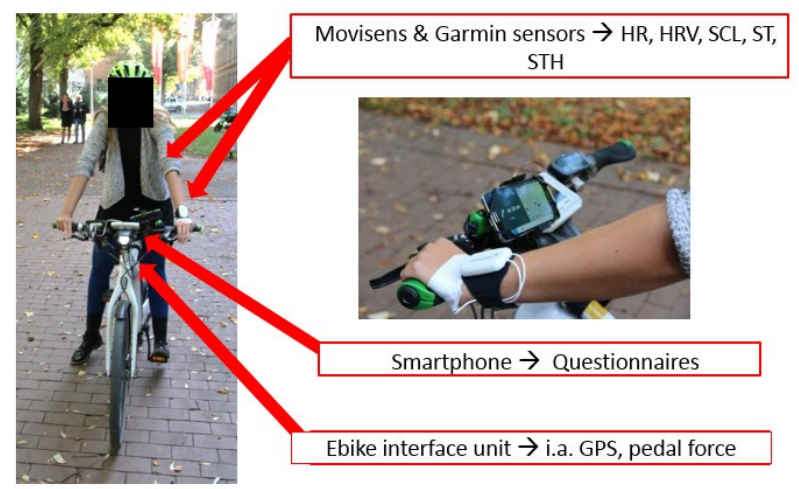

Figure 2. Proband with measurement instruments

\subsection{Personal Statistics}

Besides the sensors, personal information was added to the input data such as gender, age, height and weight.

\section{USE CASE STUTTGART}

Our use case is based in the city of Stuttgart located in southwest Germany. Like most cities, Stuttgart suffers from heavy traffic. Furthermore, as Stuttgart is located in a valley basin it struggles with increasing harmful particulate matter emission (Schweisfurth, 2018). To address this, the German government initiated versatile and diverse research projects regarding mobility in Stuttgart like The Citizen Rickshaw, or The Rotating Cargo Bike (Reallabor für Nachhaltige Mobilitätskultur, 2018). One of the newly supported project is i_city. The main target of the project is to shift more people from using unsustainable modes of transport like cars to pedelecs.

i_city (2017-2020) is a joint research venture between University of Applied Sciences Stuttgart, various municipalities and industrial partners. The focus is developing solutions central to societal challenges of sustainable city development. Six fields of action are involved: architecture, energy, finance, IT, mobility and urban planning. Amongst other things, solutions to reduce particulate matter are studied and approaches for urban and rural areas are researched.

In comparison with other sustainable modes of transport like bicycles, which require a certain amount of fitness due to the local hilly ground, pedelecs can reduce that problem. In addition, pedelecs can provide an increase in comfort for longer journeys. By using them, traffic jams can be bypassed. According to Reed and Kidd (2019), in Stuttgart the average citizen spend in a traffic jam was 108 hours in 2018 . For solely 
the last kilometre, 2.5 minutes were spent. Despite pedelec sharing stations being available, citizen prefer the car to the pedelec. This work aims at limiting the reasons why pedelecs are unheeded within the city boundaries of Stuttgart. Our approach is to detect emotions of pedelec drivers to locate areas or situations of discomfort.

To get familiar with the pedelec and the instruments, the probands first took a test drive accompanied by an investigator in the Stadtgarten nearby the university campus.

The routing was done by a navigation app and the test drives were organised in two parts. The first part represented the use of a regular bike by restricting changes in motor level support. Experiences with pedelecs were produced in study 2, as participants were not restrained in using motor support. In a large part of study 1 participants completed the test track on their own, while being monitored by the investigator with their GPS location on Google Maps. The investigator helped via the phone with technical problems. Due to safety concerns of the participants, in study 2 an investigator was following the proband on a second pedelec in visible distance

The study was set up as a combination of different situations, which should cause physical strain and psychological stress. Stuttgart is famous for its hilly structure and lots of traffic. Thus, the two factors were included to simulate a real situation of commuting. To integrate that the test track contained uphill and downhill route sections. The pavement was different, and the size of the streets varied. Some streets were very narrow with a speed limit of $30 \mathrm{~km} / \mathrm{h}$; others were multilane where the cars drove faster than $50 \mathrm{~km} / \mathrm{h}$. Moreover, one section was set through a park.

Throughout the track, there were twelve survey points. On these, the probands took a break and answered the questions. The questionnaire aimed to capture the explicit emotional level.

\subsection{Dataset}

We gathered the data of 32 female and 5 male business psychology students in total. 19 female and 3 male students took part in study 1 . In study 2, 13 female and 2 male students participated. The mean age was (22), the mean height (173) $\mathrm{cm}$ and the mean weight (65) Kg. Missing data was replaced by the method of linear interpolation. As methods of analysis, both, explanatory and predictive approaches were chosen.

\section{ARTIFICIAL INTELLIGENCE FRAMEWORK}

Data science is a discipline that supports the extraction of knowledge and unseen patterns from data. However, managing data is a tedious task. This task becomes even more complicated when we need to integrate and analyse large volume of heterogeneous data in real-time. In order to address these issues, a data processing engine that is capable of parallel data processing and supports general-purpose programming languages is the need of the hour. Apache Spark (Spark, 2019). has shown a lot of promise in this regards.

Apache Spark is a unified analytics engine for large-scale data processing. It is made up of 4 core independent libraries, namely; $\mathbf{O} \mathrm{SQL}, 2 \mathrm{MLI}$ ib for machine learning, $3 \mathrm{GraphX}$, and 4 Spark Streaming. Each of these libraries can be combined seamlessly in an application to develop a fully integrated solution. In our application, we are using the combination of MLlib and Spark Streaming library. A key advantage of the MLlib is the support of ML Pipelines. This makes it easier to combine multiple algorithms into a single pipeline. A Pipeline specifies a sequence of stages, and each stage is either a Transformer or an Estimator. These stages are run in a defined sequence; as a result, input data is transformed as it passes through each stage. A chain of multiple Transformers and Estimators constitute a ML workflow.

\subsubsection{Classification Algorithm}

As Explained in Section 2, the multi-dimensional complexity in categorizing emotions makes it difficult to define specific rules. Hence, there is a need to identify patterns and classify them using data driven techniques. Spark MLlib supports various classification methods, namely; ( binary classification, 2 multiclass classification and $\mathbf{3}$ regression. In our approach, we will use multiclass classification, as it will help us to detect complex non-linear relationship that self-adapt based on measured data.

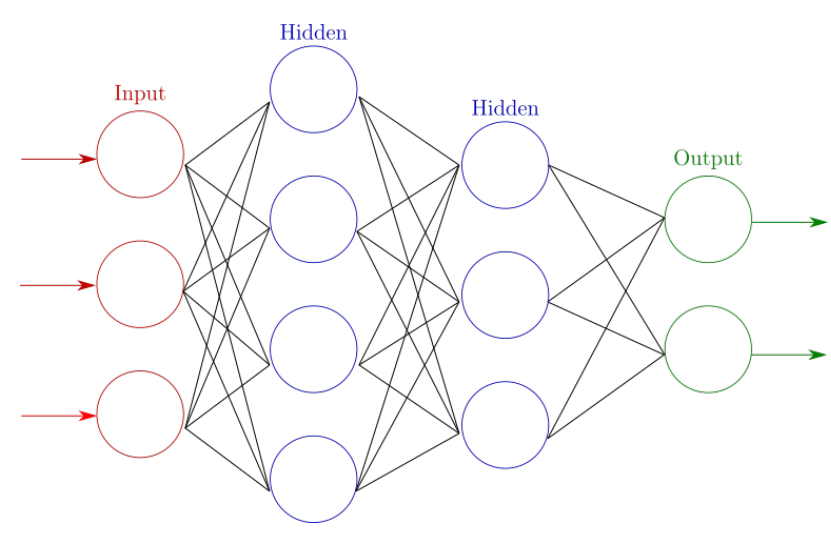

Figure 3. Multilayer Perceptron

Multilayer perceptron Classifier is a fully interconnected network of nodes called neurons. As illustrated in Figure 3 each node in the network is connected to every node in the next and previous layer. Further, each node in the network is assigned weights and connected by an output function called activation function. The activation function produces a nonlinear output for the sum of all the inputs to the node. The resultant network represents a model of nonlinear mapping between an input and an output. A typical multilayer perceptron have an input layer, one or more hidden layers and finally an output layer. A multilayer perceptron learns through a process called training. During training, the network is fed with input data and the weights in the network are adjusted until the input is mapped to the desired output. A key advantage of multilayer perceptron over statistical techniques it that it makes no prior assumptions concerning the distribution of data. Furthermore, the model can be trained to accurately generalize unseen data. These advantages of multilayer perceptron makes it an ideal candidate for emotion detection in a mobile environment. 
Decision tree classifier follows a classification strategy that resembles a tree diagram. The key to the approach is breaking up a complex decision into a several multistage simpler decisions. A typical decision tree consists of three types of nodes, namely $\mathbf{0}$ a root node, 2 one or more interior nodes, and $\mathbf{3}$ one or more terminal nodes. The root node and interior nodes are collectively called as nonterminal nodes and are directly responsible for different decision stages. Each decision stage is also referred to as a layer. Decision rules for performing the classification is performed with feature at each layer. Hence, the resultant terminal nodes represent the final classifications.

\subsection{Data \& model preparation}

Our original dataset consisted of the following input: weight, height, gender, age, body temperature at the ST, heart rate, heart rate variability, body temperature at the STH, skin conductance. To prepare the input data for training and testing we did a randomly split in 60:40 ratio. The former split was used as training data while the latter split was used to test the accuracy of the model. Furthermore, to train a ML algorithm and create a model the input data needs to transformed as label and features. To achieve this, we used ML Pipeline support. As Shown in Figure 4 our $M L$ workflow consisted of String Indexer and Vector Assembler as transformer and Multilayer Perceptron and Decision Tree as estimator.

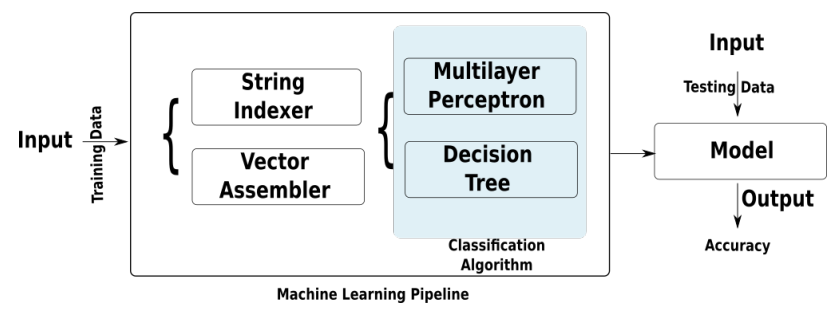

Figure 4. Machine Learning Workflow

The String Indexer encodes a string column of labels to a column of label indices. While the Vector Assembler combines a given list of columns into a single vector column. It is useful for combining raw features and features generated by different feature transformers into a single feature vector. The output of String Indexer i.e. label and Vector Assembler i.e. features is set as input for the estimator. The whole chain produces a model, which can then be used to classify unseen data.

\section{RESULTS}

In order to classify and identify different human emotions we rely on the dataset integrated from different sensors. As explained in Section 3 we have used a combination of biosensors and probands personal statistics. In the context of this paper we tried to predict only two different emotions namely, HAPPY and FEAR. The task was to predict different emotional levels of as shown in table below:

\begin{tabular}{|l|l|}
\hline Level & Happy/Fear \\
\hline 1 & not at all \\
\hline 2 & a little \\
\hline 3 & to some degree \\
\hline 4 & a lot \\
\hline 5 & extremely \\
\hline
\end{tabular}

Table 1: Level of Emotion

The table below shows the test accuracy of the predictions for two different emotions.

\begin{tabular}{|l|l|l|}
\hline Emotion & Decision Tree & $\begin{array}{l}\text { Multilayer } \\
\text { Perceptron }\end{array}$ \\
\hline Happy & $69.84 \%$ & $68.06 \%$ \\
\hline Fear & $85.82 \%$ & $84.24 \%$ \\
\hline
\end{tabular}

Table 2: Test Accuracy

The emotions happy and fear can be predicted with a high probability. However, the emotion fear has a significant higher predictions value in both classifiers. One reason is that the sensor data collated to fear is more distinct. Furthermore, in questionnaires probands could state the emotion of fear more clearly than happiness. The state of happiness is not that explicit and more mixed with related emotions such as contented, excited or elated. The feedback of the probands as well as the sensor data regarding happiness were more mixed compared to fear.

\section{DISCUSSION}

Emotion recognition is a strongly discussed topic even in the laboratory environment. However, outdoors much more parameters may trigger emotions or change the emotional state. In addition, the aggravating circumstances on a mobile vehicle during a physical exercise has to be taken into consideration. Hence, our first approach was to recognize solely the very basic emotions fear and happiness.

Moreover, fear and happiness are fit for a particular purpose. In combination with GPS data, these emotions allows specifying areas of unwellness and well-being. On one side, this kind of information may support city planners to investigate the areas of unwellness for pedelec drivers and eliminate their causes. On the other side areas of well-being may be copied and extended.

Nevertheless, much more information needs to be taken into consideration to increase the probability of the results. Outdoors the weather play a decisive role. Besides further locationdependent information can be integrated such as accident statistics or terrain information as foliation. In addition, emotions can be conveyed from current local social media posts. Particularly if the emotion correlation to an area and its extend is not obviously unambiguous, the classification algorithms may discover a correlation.

Notably is also the selection of probands. The group of probands in this study was very homogenous. Advantageous is a versatile group with different gender, age, level of fitness, height and weight. Mentionable is that all probands should be nonlocal to the test route as experiences of known locations may trigger non-relevant emotions. 


\section{OUTLOOK}

In this first approach, the study is meant to be a proof-ofconcept. A multi-modal implementation should be used in future. The current sensor data combined with face or voice recognition may provide results that are more precise. In addition, information about weather condition, outside temperature, light and sound intensity should be collected and processed.

The next step is to visualize the information in a $2 \mathrm{D}$ map highlighting the areas of interest. By virtue of the amount of the collected data, it is crucial to provide the data and results in an easy to understand way. That will provide a fast access and comprehension to the information. Not until then the results can be handled as a tool for urban planners.

Worthwhile is a close cooperation with the local municipalities. During an iterative process of improvements in urban development and continuous emotion recognition, the progress could be monitored and measured. The effect of building activities could be discovered and contribute to new insights. Thereby experiences and best practices could be shared with other cities as well as the collected data as open data for the training process of the classifier algorithms. By participating all involved parties would help optimizing the algorithm and benefiting from improved results.

\section{ACKNOWLEDGEMENTS}

This work has been jointly developed in the project Simstadt 2.0 (Funding number: 03ET1459A) and i_city (Funding number: 03FH9I01IA). The project i_city is supported by the German Federal Ministry of Education and Research (BMBF), while project Simstadt 2.0 is supported by the German Ministry of Economics (BMWi). The authors are responsible for the content of this publication. The authors would also like to thank the anonymous reviewers who have helped to increase the quality of the paper.

The project team would like to thank Prof. Dr. Volker Coors of the Dept. of Geo-Informatics as well as Prof. Dr. Thomas Bäumer and Prof. Dr. Patrick Müller of the Department of Business Psychology, HFT - University of Applied Sciences Stuttgart for their support and encouragement. Furthermore, the authors would like to express gratitude to the project team of Daimler TSS for their aid and assistance for providing and setting up the pedelecs as well as for sharing their expertise and experience.

\section{REFERENCES}

Adli, M. (2017) Stress and the city, pp 169-175, C. Bertelsmann Verlag, ISBN-10: $357010270 \mathrm{X}$

Spark, Apache. "Apache spark." (2019). Available at: https://spark.apache.org/, (Accessed: 23/05/2019).

Barrett, L. F. and Russell, J. A. (1999) 'Core affect, prototypical emotional episodes, and other things called emotion: Dissecting the elephant', Journal of Personality and Social Psychology, 76(5), pp. 805-819. doi: 10.1037/0022-3514.76.5.805.
Beck, J. (2015) Hard feelings: Science's struggle to define emotion, The Atlantic. Available at: https://www.theatlantic.com/health/archive/2015/02/ hardfeelings-sciences-struggle-to-define-emotions/385711/, (Accessed 23/07/2019).

Campbell, N. A. and Reece, J. B. (2002) Biology. Benjamin Cummings, ISBN-10: 9780805366242

Celeghin, A., Diano, M., Bagnis, A., Viola, M. and Tamietto, M. (2017) 'Basic emotions in human neuroscience: Neuroimaging and beyond', Frontiers in Psychology, 8(AUG), pp. 1-13. doi: 10.3389/fpsyg.2017.01432.

Csikszentmihalyi M., Larson R. (2014) Validity and Reliability of the Experience-Sampling Method. In: Flow and the Foundations of Positive Psychology. Springer, Dordrecht, ISBN-10: 9401790876

Delgado-Rico, E., Río-Valle ,JS, González-Jiménez, E., Campoy, C., Verdejo-García, A."BMI predicts emotion-driven impulsivity and cognitive inflexibility in adolescents with excess weight." Obesity 20.8 (2012): 1604-1610.

Geven, A., Tscheligi, M. and Noldus, L. (2009) 'Measuring Mobile Emotions: Measuring the Impossible?', pp. 5-7. In: MobileHCI '09 Proceedings of the 11th International Conference on Human-Computer Interaction with Mobile Devices and Services, doi: 10.1145/1613858.1613983

Harrison, N. A., Kreibig, S. D. and Critchley, H. D. (2013) 'A Two-Way Road', in Armony, J. and Vuilleumier, P. (eds) The Cambridge Handbook of Human Affective Neuroscience. Cambridge: Cambridge University Press, pp. 82-106. doi: 10.1017/CBO9780511843716.006.

William, J. II. 'What Is An Emotion ?', Mind, Volume os-IX, Issue 34, 1 April 1884, Pages 188-205, doi:10.1093/mind/osIX.34.188

Kohn, L. (2017) 'Erfassung emotionalen Erlebens von E-Bike Fahrenden mittels physiologischer Marker: eine Validierungsstudie im Forschungsprojekt ,i_city“ der Hochschule für Technik Stuttgart in Kooperation mit Daimler TSS'.

Kohn, L., Dastageeri, H., Bäumer, T., Moulin, S., Müller, P., and Coors, V.: Hot Or Not - Identifying Emotional "Hot Spots" In The City, ISPRS Ann. Photogramm. Remote Sens. Spatial Inf. Sci., IV-4/W7, 67-73, https://doi.org/10.5194/isprs-annalsIV-4-W7-67-2018, 2018

Landeshauptstadt Stuttgart (2019) Elektromobilität. Available at: https://www.stuttgart.de/elektromobilitaet, (Accessed: 23/05/2019).

Reallabor für Nachhaltige Mobilitätskultur. (2018) Stuttgart in Bewegung: Berichte von unterwegs. Berlin: jovis. doi: http://dx.doi.org/10.18419/opus-10234.

Reed, T. and Kidd, J. (2019) 'Global Traffic Scorecard', Inrix Research, 1 (February), pp. 1-22. doi: $10.1163 / 156854108783360159$.

Rodrigues da Silva, Antônio Nélson, Peter Zeile, Fabíola de Oliveira Aguiar, Georgios Papastefanou and Benjamin 
Sebastian Bergner. "Smart Sensoring and Barrier Free Planning: Project Outcomes and Recent Developments." Technologies for Urban and Spatial Planning: Virtual Cities and Territories. IGI Global, 2014. 93-112. Web. 23 Jul. 2019. doi:10.4018/978-14666-4349-9.ch005

Spatial Planning: Virtual Cities and Territories (pp. 93-112). Hershey PA: IGI Global., doi: 10.4018/978-1-4666-4349-9

Stockhausen, C. (2017) 'Combining Physiological Data and Context Information as an Input for Mobile Applications', Available at: http://publikationen.ub.uni-frankfurt.de/files/ 44384/ Dissertation_ClaudiaStockhausen.pdf

Rohrmann, S. and Hopp, H. (2008). Cardiovascular indicators of disgust. International journal of psychophysiology: official journal of the International Organization of Psychophysiology, 68 (3), 201-208. https://doi.org/10.1016/j.ijpsycho.2008.01.011

Rowe, D., Sibert, J. and Irwin, D. (1998) 'Heart rate variability: Indicator of user state as an aid to human-computer interaction', Proceedings of the SIGCHI Conference on Human Factors in Computing Systems, pp. 480-487. doi: 10.1145/274644.274709.

Sammito, S., Thielmann, B., Seibt, R., Klussmann, A., Weippert, M. \& Böckelmann, I. (2014). Nutzung der Herzschlagfrequenz und der Herzfrequenzvariabilität in der Arbeitsmedizin und Arbeitswissenschaft. (002/042-S2k)Leitlinie.

Schneidermeier, T. (2009) 'Usability of Mobile Devices', Opus Bayern de, p. 142. Available at: http://www.opus-bayern.de/uniregensburg/volltexte/2009/1346/pdf/Magisterarbeit_Usability_o f_Mobile_Devices_Tim_Schneidermeier.pdf

Schweisfurth, H. (2018) 'Gesundheitsrisiken durch Feinstaub und Stickstoffoxide', Atemwegs- und Lungenkrankheiten, 44(07), pp. 340-349. doi: 10.5414/atx02295.

Sherwood, L. (2007) Human physiology : from cells to systems. Thomson/Brooks/Cole., ISBN-10: 9780495014850

Siegel, E. H., Noortgate, W., Quigley, K., Sands, M., Condon, P., Chang, Y., Dy, J., Barrett, L. (2018) 'Emotion fingerprints or emotion populations? A meta-analytic investigation of autonomic features of emotion categories.', Psychological Bulletin, 144(4), pp. 343-393. doi: 10.1037/bul0000128.

Thanapattheerakul, T., Amoranto, J., Mao, K. Chan, J. (2018) 'Emotion in a Century: A Review of Emotion Recognition', pp. 1-8. doi: $10.1145 / 3291280.3291788$.

Vesterinen, V., Häkkinen, K., Hynynen, E., Mikkola, J., Hokka, L., Nummela, A. (2013) 'Heart rate variability in prediction of individual adaptation to endurance training in recreational endurance runners', Scandinavian Journal of Medicine \& Science in Sports. Society of Exploration Geophysicists, 23(2), pp. 171-180. doi: 10.1111/j.1600-0838.2011.01365.x.

VDI/VDE Innovation + Technik $\mathrm{GmbH}$, What is electric mobility? What types of vehicles does it include? (2016), Available at: https://www.erneuerbar-mobil.de/en/node/970 (Accessed: 23/05/2019).
WHO. World Health Organization, 'Healthy Cities Vision' (2019). Available at: http://www.euro.who.int/en/healthtopics/environment-and-health/urban-health/who-europeanhealthy-cities-network/healthy-cities-vision 23/05/2019).

(Accessed:

WHO. World Health Organization, 'Global Urban Ambient Air Pollution Database' (update 2016) (2017), Available at: https:/www.who.int/phe/health topics/outdoorair/databases/citi es/en/ (Accessed: 22/05/2019).

WHO. World Health Organization, 'WHO releases country estimates on air pollution exposure and health impact' (2016). Available at: https:/www.who.int/news-room/detail/27-092016-who-releases-country-estimates-on-air-pollutionexposure-and-health-impact (Accessed: 22/05/2019).

Wilhelm, J., Broschart, D. and Zeile, P. (2015) 'Potenziale von EmoMapping in der räumlichen Planung', Real Corp, pp. 261270.

Yarkoni, T. and Westfall, J. (2017) 'Choosing Prediction Over Explanation in Psychology: Lessons From Machine Learning', Perspectives on Psychological Science, 12(6), pp. 1100-1122. doi: $10.1177 / 1745691617693393$. 